\title{
Stability Study of a Permanent Magnet Synchronous Motor Sensorless Vector Control System Based on Extended EMF Model
}

\author{
Mineo Tsuji ${ }^{* a)}$ \\ Senior Member, \\ Kazuya Kojima* \\ Student Member \\ Glanny M. Ch. Mangindaan* Student Member, \\ Daisuke Akafuji* Student Member \\ Shin-ichi Hamasaki* Member
}

(Manuscript received March 14, 2012, revised Aug. 3, 2012)

\begin{abstract}
A simplified sensorless vector control system is derived by using the extended electromotive force (EMF) model. By using a steady-state voltage equation approximately, the rotor speed and position are computed from the output voltage of a $\gamma$-axis proportional plus integral (PI) current controller with decoupling control. A linear model is proposed for the small perturbation around a steady-state operating point. The system stability is discussed in terms of the trajectories of the system matrix eigenvalues for speed estimation and control parameters. The comparison between the simulation results obtained using a nonlinear model and the experimental results validates the derived system.
\end{abstract}

Keywords: permanent magnet synchronous motor (PMSM), sensorless vector control, extended EMF, stability analysis

\section{Introduction}

Because of high efficiency characteristics, permanent magnet synchronous motors (PMSM) are used in many applications. Sensorless vector control that does not use any position sensors is studied and various methods have been proposed $^{(1)}$. Sensorless control method using the extended electromotive force (EMF) model in rotating reference frame is attractive for its functional structure and can be applied to interior magnet-type motor ${ }^{(2)(3)}$. In these papers, a disturbance observer is used to compute the extended EMF. On the other hand, a simplified sensorless vector control for electrical household appliances has been proposed by using the extended EMF model ${ }^{(4)}$. Control structure is simplified by eliminating the speed and current proportional plus integral (PI) controllers and the extended EMF is computed by neglecting derivative term without using the disturbance observer. Another simplified method which does not use any motor constants is proposed ${ }^{(5)}$. In this method, the output voltage of $d$ axis PI current controller is used to compute the rotor speed and position. A similar method which uses the output voltage of $d$-axis PI current controller is reported, but it is limited to non-salient $\mathrm{PMSM}^{(6)}$.

In this paper, a simplified sensorless vector control system is derived by using the extended EMF model and a steadystate voltage equation approximately. The output voltage of $\gamma$-axis PI current controller with decoupling control is used to compute the rotor speed and position. A linear model is proposed for small perturbation around a steady-state operating point. The system stability is discussed in terms of the trajectories of the system matrix eigenvalues for speed

a) Correspondence to: Mineo Tsuji. E-mail: mineo@ nagasakiu.ac.jp

* Graduate School of Engineering, Nagasaki University

1-14, Bunkyo-machi, Nagasaki 852-8521, Japan estimation and control parameters. Experimental system is constructed by a digital signal processor (DSP) based PWM inverter drive. The comparison between simulation results obtained using a nonlinear model and the experimental results shows validity and usefulness of the proposed models and the derived system.

\section{Sensorless System}

The definition of $d-q$ axis and $\gamma-\delta$ axis studied in this paper is shown in Fig. 1. The $\gamma-\delta$ axis rotates synchronously with an estimated magnetic pole's angle.

The $\gamma-\delta$ axis equation of interior permanent magnet synchronous motor (IPMSM) is described by using extended EMF as follows ${ }^{(2)}$ :

$$
\left[\begin{array}{c}
v_{\gamma} \\
v_{\delta}
\end{array}\right]=\left[\begin{array}{cc}
R_{s}+p L_{d} & -\omega_{r} L_{q} \\
\omega_{r} L_{q} & R_{s}+p L_{d}
\end{array}\right]\left[\begin{array}{c}
i_{\gamma} \\
i_{\delta}
\end{array}\right]+\left[\begin{array}{c}
\hat{e}_{\gamma} \\
\hat{e}_{\delta}
\end{array}\right] \ldots \ldots \ldots
$$

where,

$$
\begin{aligned}
& E_{e x}=\omega_{r}\left\{\left(L_{d}-L_{q}\right) i_{d}+\psi\right\}-\left(L_{d}-L_{q}\right) p i_{q} \cdots \cdots \\
& {\left[\begin{array}{c}
\hat{e}_{\gamma} \\
\hat{e}_{\delta}
\end{array}\right]=E_{e x}\left[\begin{array}{c}
\sin \theta_{e} \\
\cos \theta_{e}
\end{array}\right]+\left(\hat{\omega}_{r}-\omega_{r}\right) L_{d}\left[\begin{array}{c}
-i_{\delta} \\
i_{\gamma}
\end{array}\right] \ldots \ldots \ldots \ldots}
\end{aligned}
$$

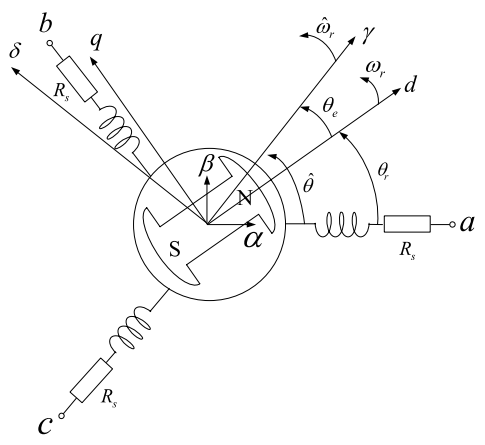

Fig. 1. Definition of $d-q$ axis and $\gamma-\delta$ axis 


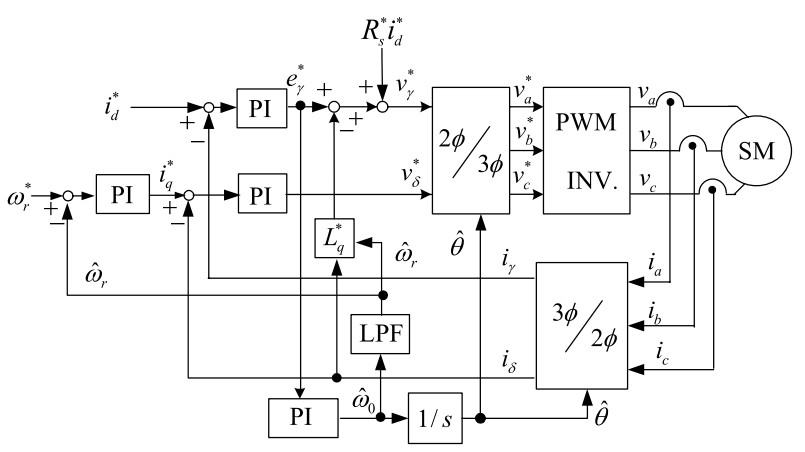

Fig. 2. Simplified sensorless control system based on extended EMF

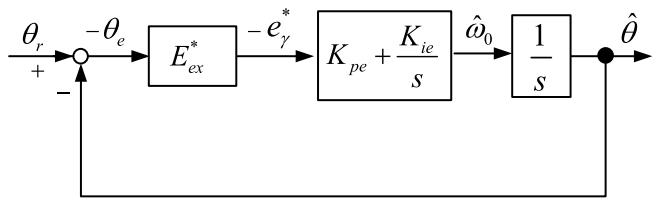

Fig. 3. Approximate block diagram of speed estimation

The actual error angle $\theta_{e}$ shown in Fig. 1 is defined as

$$
\theta_{e}=\hat{\theta}-\theta_{r}
$$

Conventional sensorless vector control method estimates $\gamma-\delta$ extended EMF $\hat{e}_{\gamma}, \hat{e}_{\delta}$ by using a disturbance observer from (1) and computes approximate error angle $\hat{\theta}_{e}$ of (4) as follows ${ }^{(2)(3)}$ :

$$
\hat{\theta}_{e}=\tan ^{-1}\left(\frac{\hat{e}_{\gamma}}{\hat{e}_{\delta}}\right)
$$

The rotor speed is computed by PI controlling $\hat{\theta}_{e}$ so as to converge to zero ${ }^{(2)(3)}$.

In this paper, we derive a simpler method without using the disturbance observer. We compute the $\gamma$-axis extended EMF by neglecting derivative term in (1) as follows:

$$
e_{\gamma}^{*}=v_{\gamma}^{*}-R_{s}^{*} i_{d}^{*}+\hat{\omega}_{r} L_{q}^{*} i_{\delta}
$$

From (5) and (6), we estimate the rotor angular speed by

$$
\hat{\omega}_{0}=-\left(K_{p e}+\frac{K_{i e}}{s}\right) e_{\gamma}^{*}
$$

In order to reduce high frequency ripple, a low pass filter is used to compute the rotor speed as follows:

$$
\hat{\omega}_{r}=\frac{\omega_{c}}{s+\omega_{c}} \hat{\omega}_{0}
$$

The sensorless vector control system derived in this paper is shown in Fig. 2. Fig. 3 shows an approximate block diagram of speed and rotor angle estimation. The transfer function of rotor angle is expressed as

$$
\frac{\hat{\theta}}{\theta_{r}}=\frac{E_{e x}^{*} K_{p e} s+E_{e x}^{*} K_{i e}}{s^{2}+E_{e x}^{*} K_{p e} s+E_{e x}^{*} K_{i e}}
$$

where,

$$
E_{e x}^{*}=\hat{\omega}_{r}\left\{\left(L_{d}-L_{q}\right) i_{d}^{*}+\psi\right\}
$$

By using damping coefficient $\zeta$ and natural angular frequency $\omega_{n}$, the PI control gains are expressed as

$$
K_{p e}=\frac{2 \zeta \omega_{n}}{E_{e x}^{*}}, \quad K_{i e}=\frac{\omega_{n}^{2}}{E_{e x}^{*}}
$$

\section{Analysis of Sensorless System}

3.1 Nonlinear Model In order to analyze the system shown in Fig. 2, we choose the $d-q$ axis and $\gamma-\delta$ axis of Fig. 1 . By assuming ideal voltage control of PWM inverter, we have

$$
v_{a}=v_{a}^{*}, \quad v_{b}=v_{b}^{*}, \quad v_{c}=v_{c}^{*}
$$

Since $\gamma-\delta$ transformation of controller is the same as the analysis, the following relation is obtained.

$$
v_{\gamma}=v_{\gamma}^{*}, \quad v_{\delta}=v_{\delta}^{*}
$$

By using the actual error angle $\theta_{e}$, the $d-q$ variables are expressed by the following co-ordinate transformation.

$$
\begin{aligned}
& {\left[\begin{array}{l}
v_{d} \\
v_{q}
\end{array}\right]=\left[\begin{array}{cc}
\cos \theta_{e} & -\sin \theta_{e} \\
\sin \theta_{e} & \cos \theta_{e}
\end{array}\right]\left[\begin{array}{l}
v_{\gamma}^{*} \\
v_{\delta}^{*}
\end{array}\right]} \\
& {\left[\begin{array}{l}
i_{d} \\
i_{q}
\end{array}\right]=\left[\begin{array}{cc}
\cos \theta_{e} & -\sin \theta_{e} \\
\sin \theta_{e} & \cos \theta_{e}
\end{array}\right]\left[\begin{array}{l}
i_{\gamma} \\
i_{\delta}
\end{array}\right]}
\end{aligned}
$$

The $d-q$ state equations of PMSM are obtained by Park's equation as follows:

$$
\begin{aligned}
& p i_{d}=\frac{1}{L_{d}}\left(v_{d}-R_{s} i_{d}+\omega_{r} L_{q} i_{q}\right) \ldots \ldots \ldots \\
& p i_{q}=\frac{1}{L_{q}}\left(v_{q}-R_{s} i_{q}-\omega_{r} L_{d} i_{d}-\omega_{r} \psi\right) \cdots \\
& p \omega_{r}=\frac{P^{2}}{4 J}\left\{\psi i_{q}+\left(L_{d}-L_{q}\right) i_{d} i_{q}\right\}-\frac{P}{2 J} T_{L}
\end{aligned}
$$

where, $P$ is number of poles, $J$ is moment of inertia, and $T_{L}$ is load torque.

The controller is expressed by following equations.

PI speed controller:

$$
\begin{aligned}
& p w_{1}=\omega_{r}^{*}-\hat{\omega}_{r} \ldots \ldots \ldots \ldots \ldots \ldots \ldots \ldots \ldots \ldots \ldots \ldots \ldots \ldots \\
& i_{q}^{*}=K_{p s}\left(\omega_{r}^{*}-\hat{\omega}_{r}\right)+K_{i s} w_{1} \cdots \ldots \ldots \ldots \ldots \ldots \ldots
\end{aligned}
$$

$D$ axis PI controller:

$$
\begin{aligned}
& p w_{2}=i_{d}^{*}-i_{\gamma} \ldots \ldots \ldots \ldots \\
& e_{\gamma}^{*}=K_{p d}\left(i_{d}^{*}-i_{\gamma}\right)+K_{i d} w_{2}
\end{aligned}
$$

$Q$ axis PI controller:

$$
\begin{aligned}
& p w_{3}=i_{q}^{*}-i_{\delta} \ldots \ldots \ldots \ldots \\
& v_{\delta}^{*}=K_{p q}\left(i_{q}^{*}-i_{\delta}\right)+K_{i q} w_{3}
\end{aligned}
$$

PI speed estimator:

$$
\begin{aligned}
& p w_{4}=e_{\gamma}^{*} \ldots \ldots \ldots \ldots \\
& \hat{\omega}_{0}=-K_{p e} e_{\gamma}^{*}-K_{i e} w_{4}
\end{aligned}
$$

Low pass filter of speed estimation:

$$
p \hat{\omega}_{r}=-\omega_{c} \hat{\omega}_{r}+\omega_{c} \hat{\omega}_{0}
$$

By taking derivative of (4), we have

$$
p \theta_{e}=\hat{\omega}_{r}-\omega_{r}
$$

A nonlinear model of the sensorless system shown in Fig. 2 is obtained by (6) and (13)-(27). By using this model, we can compute transient responses. 
3.2 Linear Model The steady-state solutions are obtained by setting derivative operator $p$ to zero in the nonlinear model. When the stator resistance and $q$-axis inductance of (6) are correct, the steady-state actual error angle $\theta_{e 0}$ becomes zero.

The system is linearized about its equilibrium state to obtain a linear model for small signal performance evaluations, such as stability and transient response. The linearized equation of PMSM in matrix form is obtained from (15)-(17) as

$$
p \Delta \boldsymbol{x}_{s}=\boldsymbol{A}_{\boldsymbol{s}} \Delta \boldsymbol{x}_{s}+\boldsymbol{B}_{s} \Delta \boldsymbol{u}_{\boldsymbol{s}}+\boldsymbol{B}_{T} \Delta T_{L}
$$

where,

$$
\begin{aligned}
& \Delta \boldsymbol{x}_{\boldsymbol{s}}=\left[\begin{array}{lll}
\Delta i_{d} & \Delta i_{q} & \Delta \omega_{r}
\end{array}\right]^{T} \\
& \Delta \boldsymbol{u}_{s}=\left[\begin{array}{ll}
\Delta v_{d} & \Delta v_{q}
\end{array}\right]^{T}
\end{aligned}
$$

The elements of the matrices are shown in the appendix.

By taking small perturbation of (13), we have

$$
\begin{aligned}
& \Delta v_{d}=\Delta v_{\gamma}^{*}-v_{\delta 0}^{*} \Delta \theta_{e} \\
& \Delta v_{q}=v_{\gamma 0}^{*} \Delta \theta_{e}+\Delta v_{\delta}^{*}
\end{aligned}
$$

The linear state equation of controller is expressed from (18), (20), (22), (24), (26), and (27) as follows:

$$
p \Delta w=A_{w} \Delta w+A_{x} \Delta \boldsymbol{x}_{s}+B_{r} \Delta r
$$

where,

$$
\begin{aligned}
& \Delta \boldsymbol{w}=\left[\begin{array}{llllll}
\Delta \hat{\omega}_{r} & \Delta w_{1} & \Delta w_{2} & \Delta w_{3} & \Delta w_{4} & \Delta \theta_{e}
\end{array}\right]^{T} \\
& \Delta \boldsymbol{r}=\left[\begin{array}{lll}
\Delta i_{d}^{*} & \Delta \omega_{r}^{*}
\end{array}\right]^{T}
\end{aligned}
$$

The input vector is expressed by using state variables and references as

$$
\Delta \boldsymbol{u}_{\boldsymbol{s}}=\boldsymbol{F}_{w} \Delta w+\boldsymbol{F}_{\boldsymbol{x}} \Delta \boldsymbol{x}_{\boldsymbol{s}}+\boldsymbol{F}_{\boldsymbol{r}} \Delta \boldsymbol{r}
$$

For simplicity, the PI gains of speed estimator is assumed constant to derive (31) and (32). From (28), (31), and (32), we obtain a linear model of the complete system as

$$
\begin{aligned}
p\left[\begin{array}{c}
\Delta \boldsymbol{x}_{s} \\
\Delta \boldsymbol{w}
\end{array}\right]= & {\left[\begin{array}{cc}
\boldsymbol{A}_{\boldsymbol{s}}+\boldsymbol{B}_{\boldsymbol{s}} \boldsymbol{F}_{\boldsymbol{x}} & \boldsymbol{B}_{\boldsymbol{s}} \boldsymbol{F}_{\boldsymbol{w}} \\
\boldsymbol{A}_{\boldsymbol{x}} & \boldsymbol{A}_{\boldsymbol{w}}
\end{array}\right]\left[\begin{array}{c}
\Delta \boldsymbol{x}_{\boldsymbol{s}} \\
\Delta \boldsymbol{w}
\end{array}\right]+\left[\begin{array}{c}
\boldsymbol{B}_{\boldsymbol{s}} \boldsymbol{F}_{\boldsymbol{r}} \\
\boldsymbol{B}_{\boldsymbol{r}}
\end{array}\right] \Delta \boldsymbol{r} } \\
& +\left[\begin{array}{c}
\boldsymbol{B}_{\boldsymbol{T}} \\
0
\end{array}\right] \Delta T_{L} \ldots \ldots \ldots \ldots \ldots \ldots \ldots \ldots \ldots
\end{aligned}
$$

The stability and transient responses of the system shown in Fig. 2 can be studied by the linear model. We have confirmed that the transient responses obtained by the nonlinear model are in good agreement with those by (33) in the neighborhood of operating point.

3.3 Stability Analysis By using the parameters of tested PMSM, the stability is studied. For simplicity, the $d$ axis current command $i_{d}^{*}$ is set to 0 . The band width of the PI current controller is designed as $1000 \mathrm{rad} / \mathrm{s}$. Fig. 4 shows the trajectories of the poles (eigenvalues) in the $s$-plane calculated from (33), in which $\omega_{n}$ of (10) is changed from 10 to 200 and $\omega_{s c}$ is changed from 30 to 100 . Where, $\omega_{s c}$ is the gain crossover angular frequency of PI speed control. When the pole is near the imaginary axis, the system becomes oscillating. Therefore, suitable value of $\omega_{n}$ must be chosen. If

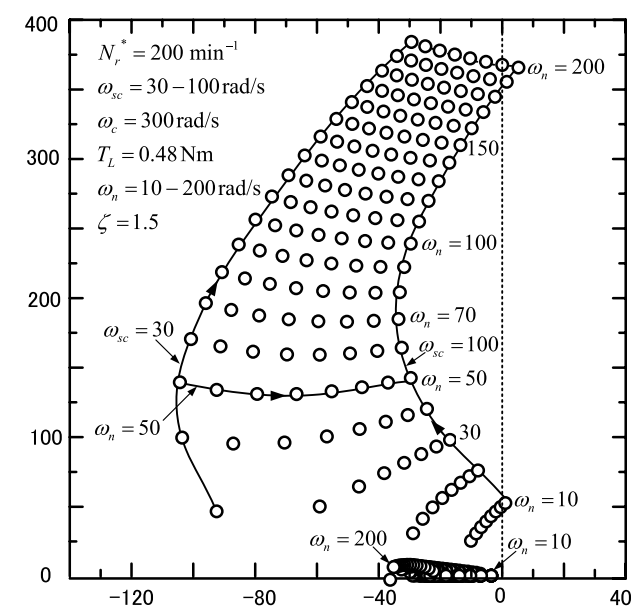

Fig. 4. Trajectories of poles for the change of $\omega_{n}$ and $\omega_{s c}$

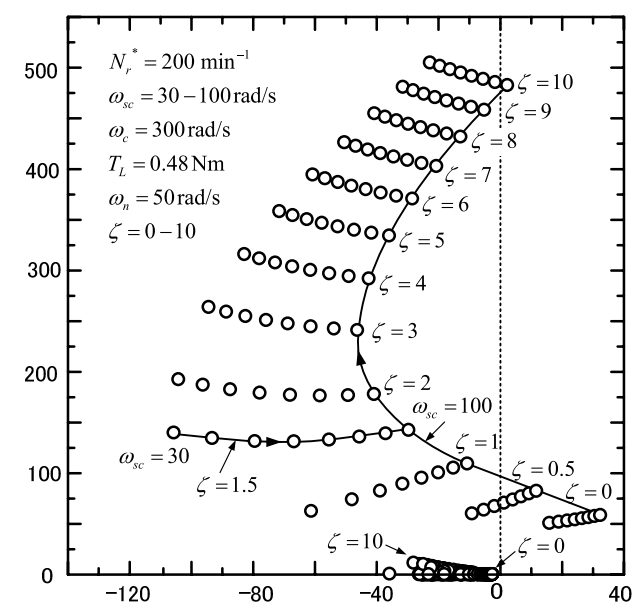

Fig. 5. Trajectories of poles for the change of $\zeta$ and $\omega_{s c}$

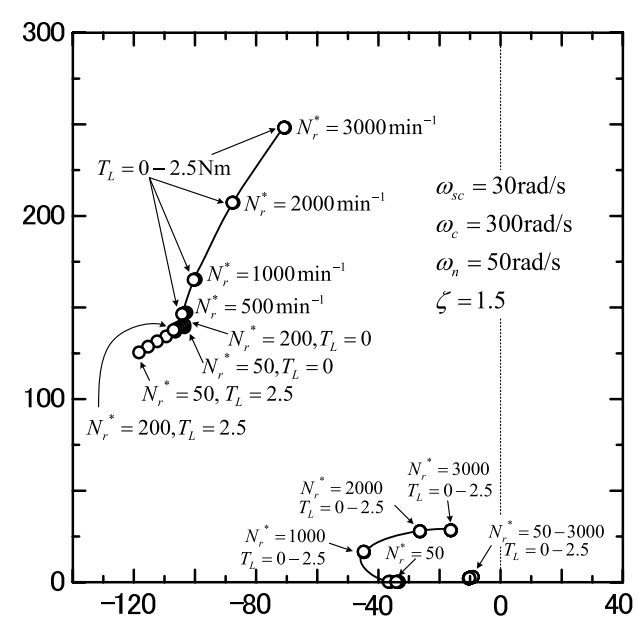

Fig. 6. Trajectories of poles for the change of speed and torque

the $\omega_{s c}$ is small, the system is more stable, however the response of speed becomes slow. Fig. 5 shows the trajectories of the poles, in which $\zeta$ of (10) is changed from 0 to 10 and $\omega_{s c}$ is changed from 30 to 100 . When $\zeta$ is small the system becomes unstable and large value of $\zeta$ leads the system to high frequency instability. Therefore, we choose as $\omega_{n}=$ 

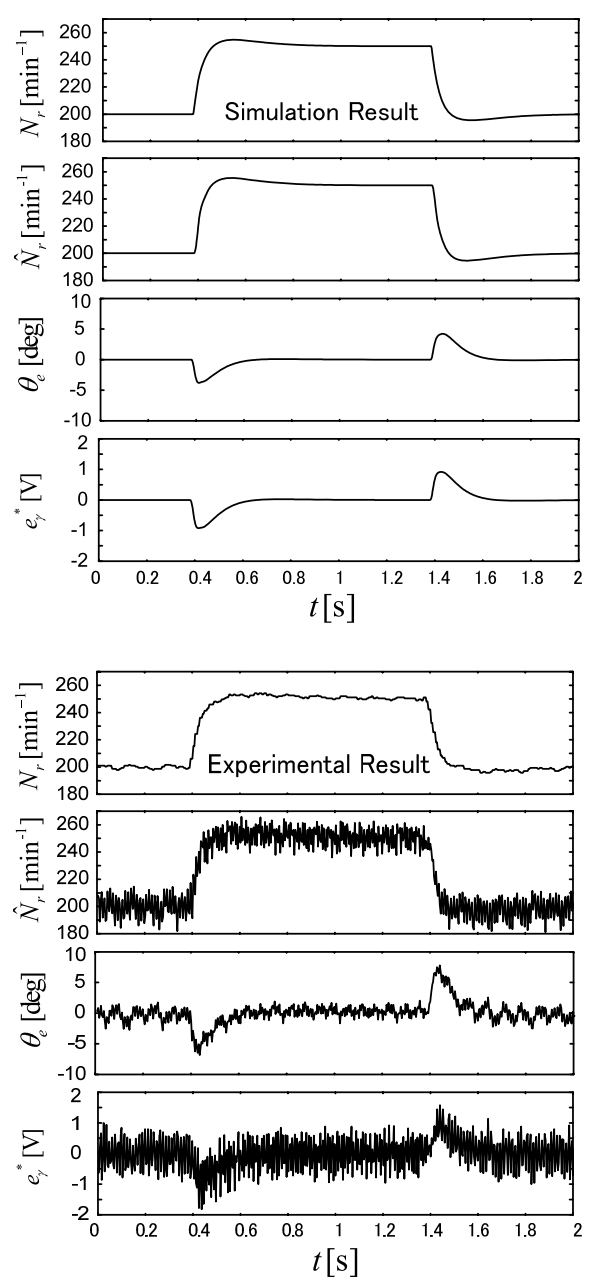

$\omega_{s c}=30 \mathrm{rad} / \mathrm{s}, \omega_{n}=50 \mathrm{rad} / \mathrm{s}, \zeta=1.5, T_{L}=0.48 \mathrm{Nm}$

Fig. 7. Transient responses for the step change of speed

50, $\zeta=1.5$, and $\omega_{s c}=30$ to ensure the system stability. By using these parameters, Fig. 6 shows the trajectories of the poles, in which speed command $N_{r}^{*}$ is varied from $50 \mathrm{~min}^{-1}$ to $3000 \mathrm{~min}^{-1}$ and the load torque $T_{L}$ is varied from 0 to $2.5 \mathrm{Nm}$. For the change of speed and load torque, the system is stable at all operating points.

\section{Experimental Results}

The control system is implemented by a DSP (TMS320 C33) based PWM inverter. The sampling period of DSP is $100 \mu \mathrm{s}$ and the switching frequency is $10 \mathrm{kHz}$. The tested PMSM has the following rated and nominal values: rated output $800 \mathrm{~W}$, rated speed $3000 \mathrm{~min}^{-1}$, rated torque $2.55 \mathrm{Nm}, P$ $=8, J=0.0048 \mathrm{kgm}^{2}$ (including DC generator), $R_{s}=0.4 \Omega$, $L_{d}=3.42 \mathrm{mH}, L_{q}=3.82 \mathrm{mH}$, and $\psi 0.1425 \mathrm{~Wb}$ which were also used in the analysis. Because the dead time and the nonideal features of IGBT influence the output voltage of the inverter, a compensating algorithm is developed for the experimental system ${ }^{(9)}$.

Figs. 7-10 show the transient responses for step change of speed command. The simulation results are obtained by solving the nonlinear model described in section 3.1. In these figures, the speed command $N_{r}^{*}$ is stepped from $200 \mathrm{~min}^{-1}$ to $250 \mathrm{~min}^{-1}$ and then down to $200 \mathrm{~min}^{-1}$. When designed
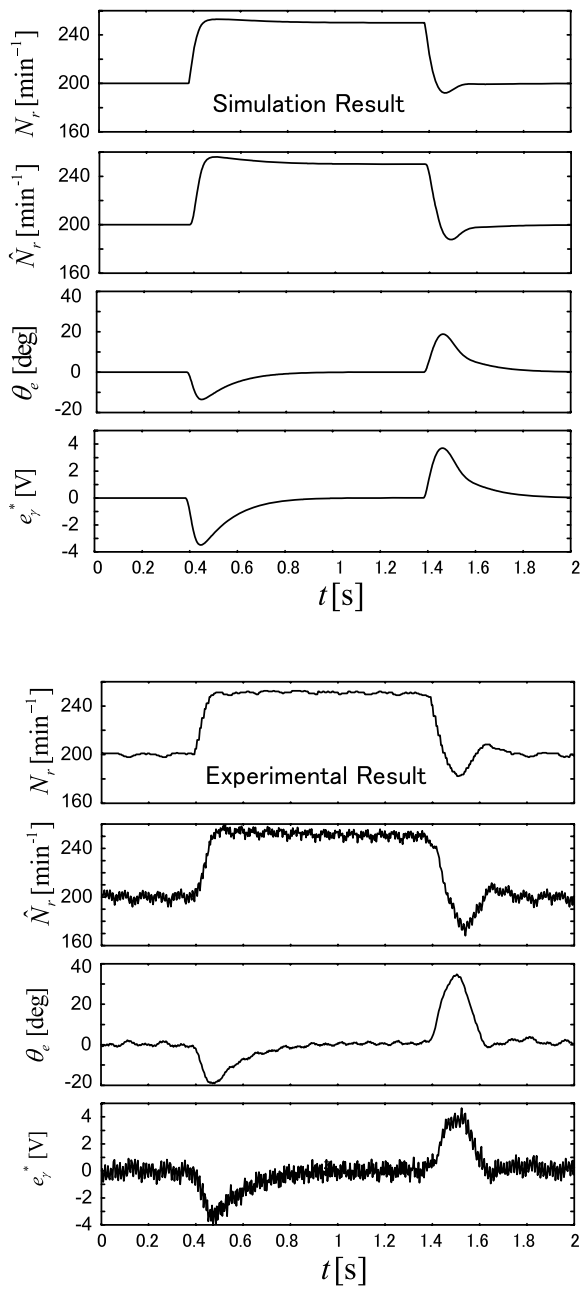

$\omega_{s c}=30 \mathrm{rad} / \mathrm{s}, \omega_{n}=20 \mathrm{rad} / \mathrm{s}, \zeta=1.5, T_{L}=0.48 \mathrm{Nm}$

Fig. 8. Transient responses for the step change of speed

parameters $\left(\omega_{n}=50, \zeta=1.5\right.$, and $\left.\omega_{s c}=30\right)$ are used, desirable responses of actual speed $N_{r}$ and estimated speed $\hat{N}_{r}$ are obtained as shown in Fig. 7. The actual error angle $\theta_{e}$ is defined as (4). When $\omega_{n}$ is small, the $\theta_{e}$ becomes large and the stability is not good as shown in Fig. $8\left(\omega_{n}=20\right)$. When $\omega_{n}$ is large, the PI speed estimation gains of (10) are large and high frequency ripples are observed in estimation speed and error angle as shown in Fig. $9\left(\omega_{n}=110\right)$. When $\zeta$ is small, the $\mathrm{P}$ gain of speed estimation is small and low frequency oscillation is observed as shown in Fig. $10(\zeta=0.5)$. The poles corresponding to the equilibrium point of Fig. 10 are shown in Fig. 5. Since the poles are close to imaginary axis in this case, it seems that the PWM operation, machine parameter change and noise which are not considered in the analysis cause the difference between simulation results and experimental ones. It is confirmed that the simulation results can predict the experimental results in all cases, except high frequency ripples.

Fig. 11 shows the experimental results when the speed command is stepped from $1000 \mathrm{~min}^{-1}$ to $1050 \mathrm{~min}^{-1}$ and then down to $1000 \mathrm{~min}^{-1}$. Fig. 12 shows the experimental results when the speed command is stepped from $2500 \mathrm{~min}^{-1}$ to $2550 \mathrm{~min}^{-1}$ and then down to $2500 \mathrm{~min}^{-1}$. The designed parameters $\left(\omega_{n}=50, \zeta=1.5, \omega_{s c}=30\right)$ are used and smooth 

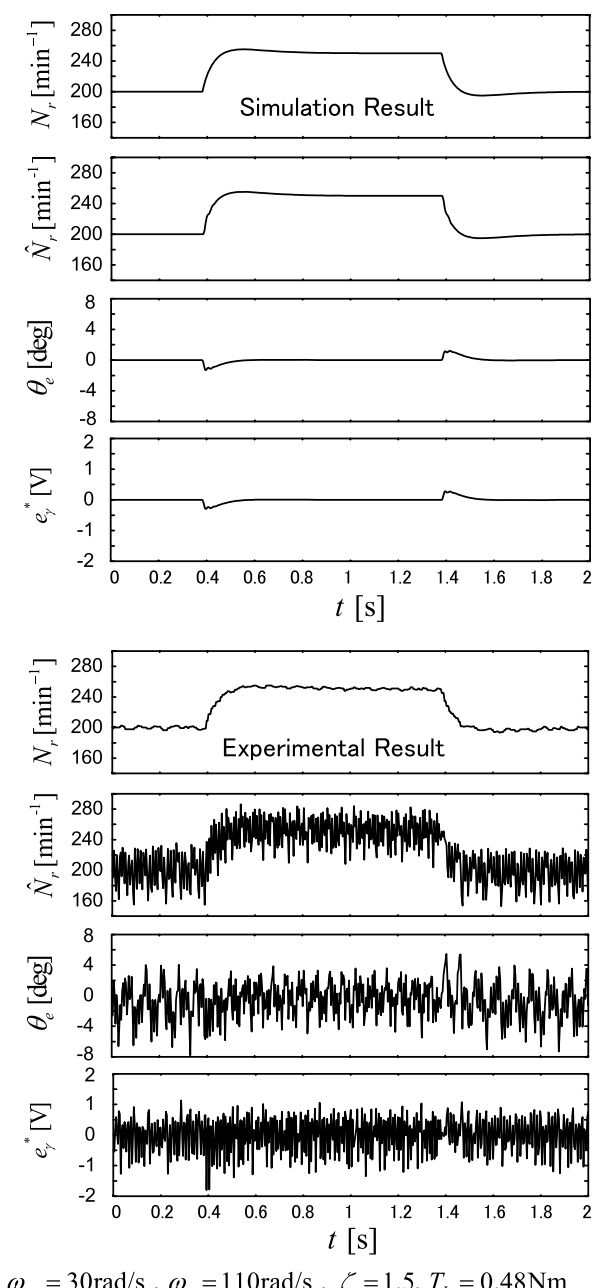

$\omega_{s c}=30 \mathrm{rad} / \mathrm{s}, \omega_{n}=110 \mathrm{rad} / \mathrm{s}, \zeta=1.5, T_{L}=0.48 \mathrm{Nm}$

Fig. 9. Transient responses for the step change of speed

control of speed is obtained in both cases. By comparing the results of Figs. 11 and 12 with those of Fig. 7, it is observed that the high frequency ripples of $\hat{N}_{r}$ and $\theta_{e}$ are decreased drastically. Since the extended EMF is large at high speed, we can estimate rotor position by small PI estimation gains. It seems that a little increase of overshoot in Fig. 12 comparing with that in Fig. 11 is caused by the poles shown in Fig. 6. The steady state experimental position error $\theta_{e}$ becomes large as shown in Fig. 12 at high speed ${ }^{(7)}$. The change of $q$-axis inductance $L_{q}$ and the PWM voltage error are considered as the reason. In order to reduce the error $\theta_{e}$, an identification of $L_{q}$ is desired. In the simulation in Fig. 12, the actual $L_{q}$ is changed ( $L_{q}^{*}$ in the controller is $3.82 \mathrm{mH}$ ). Large influence of $L_{q}$ on transient characteristics are not seen in the results. Therefore, the proposed linear model is useful for the basic estimation of stability.

Figs. 13 and 14 show the experimental results when the load torque is stepped from $0.15 \mathrm{Nm}$ to $0.88 \mathrm{Nm}$ and from $0.88 \mathrm{Nm}$ to $0.15 \mathrm{Nm}$ respectively. The load torque is changed by turning a switch of DC generator connected to PMSM between on and off. It is observed that the speed is disturbed by the change of load but it is recovered in a stable state.

\section{Conclusions}

We have derived a simplified sensorless vector control
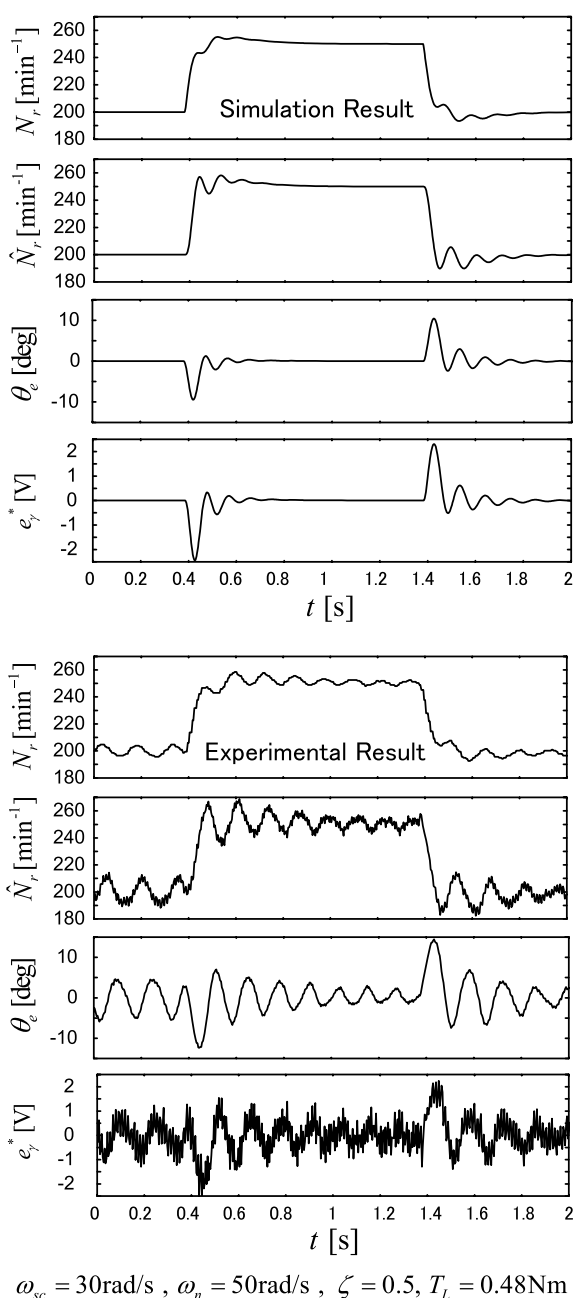

Fig. 10. Transient responses for the step change of speed
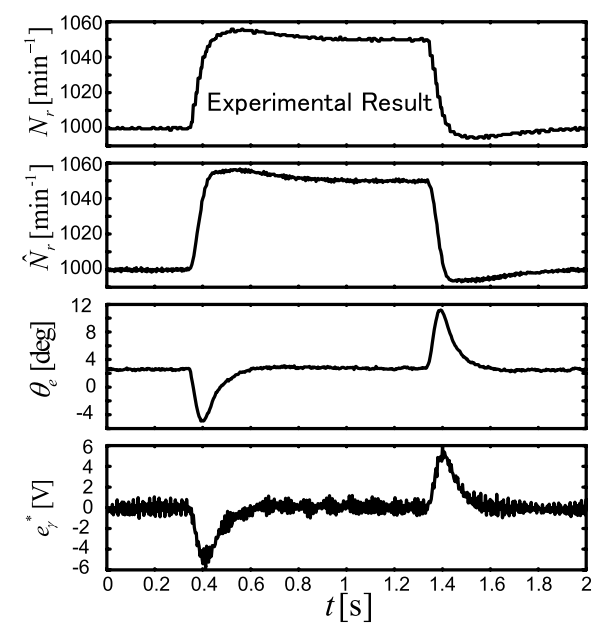

$\omega_{s c}=30 \mathrm{rad} / \mathrm{s}, \omega_{n}=50 \mathrm{rad} / \mathrm{s}, \zeta=1.5, T_{L}=0.76 \mathrm{Nm}$

Fig. 11. Transient responses for the step change of speed

system by using the extended EMF model. This system does not need any observers which are used in the standard extended EMF based method. In order to analyze the stability of the system in detail, we have proposed a linear model for small perturbation around a steady-state operating point. 

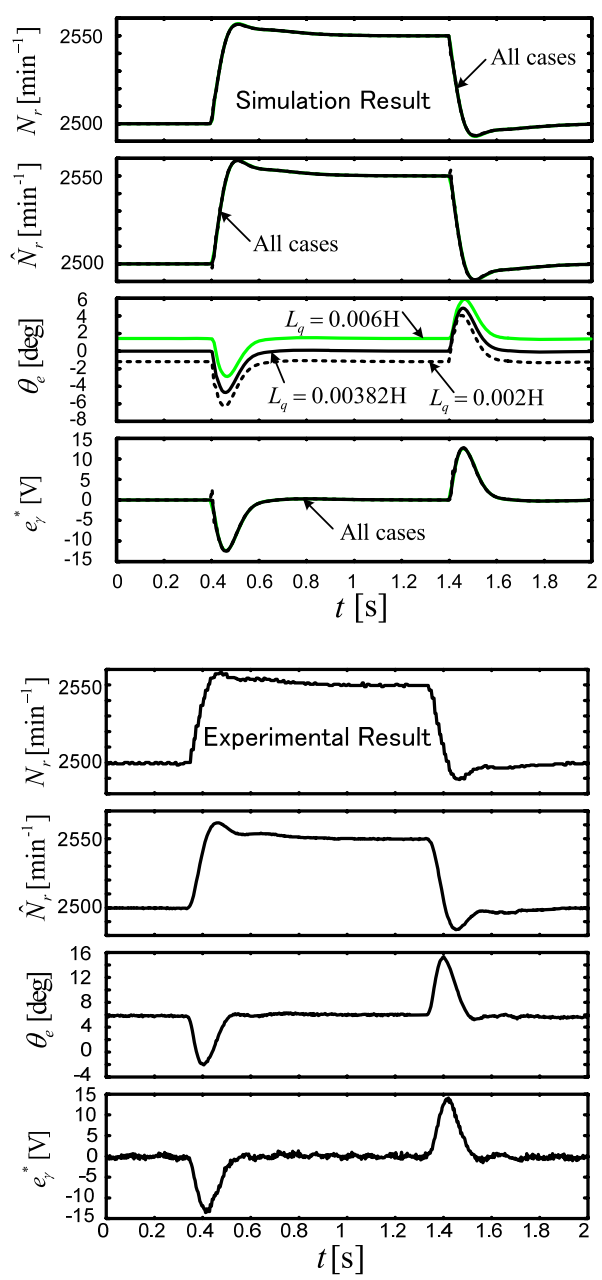

$\omega_{s c}=30 \mathrm{rad} / \mathrm{s}, \omega_{n}=50 \mathrm{rad} / \mathrm{s}, \zeta=1.5, T_{L}=0.93 \mathrm{Nm}$

Fig. 12. Transient responses for the step change of speed

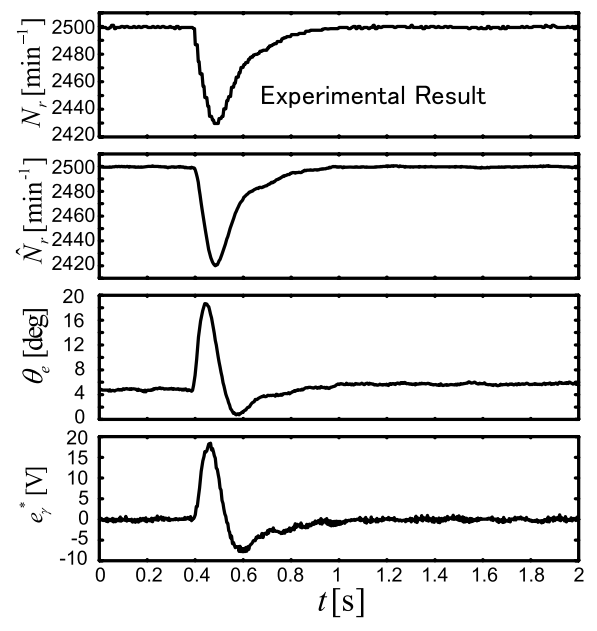

$\omega_{s c}=30 \mathrm{rad} / \mathrm{s}, \omega_{n}=50 \mathrm{rad} / \mathrm{s}, \zeta=1.5, N_{r}^{*}=2500 \mathrm{~min}^{-1}$

Fig. 13. Transient responses for the step change of load torque from $0.15 \mathrm{Nm}$ to $0.88 \mathrm{Nm}$

By the trajectories of system matrix eigenvalues, the system stability has been discussed for speed estimation and speed control parameters. Stability analysis of PMSM sensorless vector control system using linear model has not been

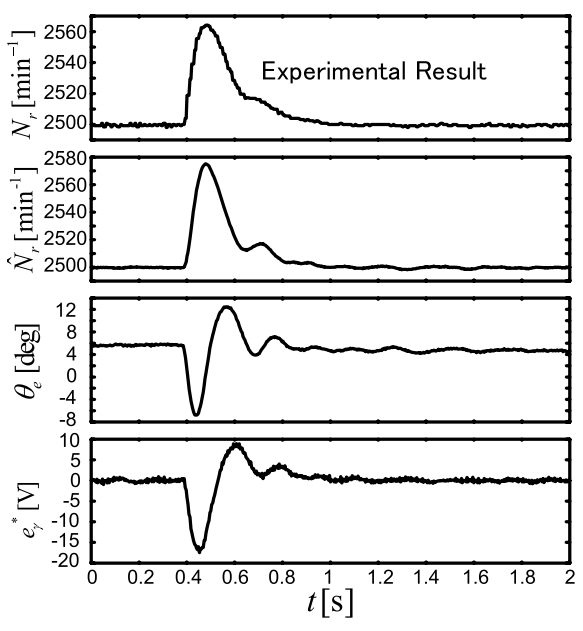

$$
\omega_{s c}=30 \mathrm{rad} / \mathrm{s}, \omega_{n}=50 \mathrm{rad} / \mathrm{s}, \zeta=1.5, N_{r}^{*}=2500 \mathrm{~min}^{-1}
$$

Fig. 14. Transient responses for the step change of load torque from $0.88 \mathrm{Nm}$ to $0.15 \mathrm{Nm}$

reported. Since proposed stability analysis using the linear model can be executed in short computation time than commercial simulation tools, it is useful for the design of controller. Experimental system is constructed by DSP based PWM inverter drive. Comparison between simulation results using a nonlinear model and experimental results shows validity and usefulness of the derived system and the proposed analytical models.

\section{References}

( 1 ) P.P. Acarnley and J.F. Watson: "Review of Position-Sensorless Operation of Brushless Permanent-Magnet Machines", IEEE Trans. Ind. Electron., Vol.53, No.2, pp.352-362 (2006)

( 2 ) Z. Chen, M. Tomita, S. Doki, and S. Okuma: "An Extended Electromotive Force Model for Sensorless Control of Interior Permanent-Magnet Synchronous Motors", IEEE Trans. Ind. Electron., Vol.50, No.2, pp.288-295 (2003)

( 3 ) S. Morimoto, K. Kawamoto, M. Sanada, and Y. Takeda: "Sensorless Control Strategy for Salient-Pole PMSM Based on Extended EMF in Rotating Reference Frame", IEEE Trans. Ind. Applicat., Vol.38, No.4, pp.1054-1061 (2002)

( 4 ) K. Sakamoto, Y. Iwaji, T. Endo, T. Taniguchi, T. Niki, M. Kawamata, and A. Kawamura: "Position Sensorless Vector Control of Permanent Magnet Synchronous Motors for Electrical Household Appliances", Proc. of the Power Conversion Conference PCC-Nagoya 2007, LS4-3-3, pp.1119-1125 (2007)

( 5 ) K. Yamanaka, T. Ohnishi, and M. Hojo: "A Novel Position Sensorless Vector Control of Permanent-Magnet Synchronous Motors", Proc. of the PCCNagoya 2007, DS8-3-1, pp.290-295 (2007)

( 6 ) J.K. Seok, J.K. Lee, and D.C. Lee: "Sensorless Speed Control of Nonsalient Permanent-Magnet Synchronous Motor Using Rotor-Position-Tracking PI Controller", IEEE Trans. Ind. Electron., Vol.53, No.2, pp.399-405 (2006)

( 7 ) M. Tsuji, K. Kojima, and S. Hamasaki: "A Method of Sensorless Vector Control using Control Voltage of IPMSM", Proc. of Annual Conference of the IEE Japan Industry Application Society, No.I-196, pp.835-838 (2011) (in Japanese)

( 8 ) M. Tsuji, S. Matsumoto, K. Kojima, and S. Hamasaki: "A Linear Model of Permanent Magnet Synchronous Motor Sensorless Control System using Extended EMF", Record of IEE Japan Symposium, SPC-10-83, pp.1-6 (2010) (in Japanese)

( 9 ) M. Tsuji, S. Chen, K. Izumi, and E. Yamada: "A Sensorless Vector Control System for Induction Motors Using $q$-Axis Flux with Stator Resistance Identification", IEEE Trans. Ind. Electron., Vol.48, No.1, pp.185-194 (2001) 


\section{Appendix}

$\boldsymbol{A}_{s}=\left[\begin{array}{ccc}-\frac{R_{s}}{L_{d}} & \frac{L_{q} \omega_{r 0}}{L_{d}} & \frac{L_{q} i_{q 0}}{L_{d}} \\ -\frac{L_{d} \omega_{r 0}}{L_{q}} & -\frac{R_{s}}{L_{q}} & -\frac{L_{d} i_{d 0}+\psi}{L_{q}} \\ a_{s 1} & a_{s 2} & 0\end{array}\right]$
$a_{s 1}=\frac{P^{2}\left(L_{d}-L_{q}\right) i_{q 0}}{4 J}, \quad a_{s 2}=\frac{P^{2}\left\{\psi+\left(L_{d}-L_{q}\right) i_{d 0}\right\}}{4 J}$

$\boldsymbol{B}_{s}=\left[\begin{array}{cc}\frac{1}{L_{d}} & 0 \\ 0 & \frac{1}{L_{q}} \\ 0 & 0\end{array}\right], \quad \boldsymbol{B}_{T}=\left[\begin{array}{c}0 \\ 0 \\ -\frac{P}{2 J}\end{array}\right]$

$\boldsymbol{A}_{\boldsymbol{w}}=\left[\begin{array}{cccccc}-\omega_{c} & 0 & -\omega_{c} K_{p e} K_{i d} & 0 & -\omega_{c} K_{i e} & \omega_{c} K_{p e} K_{p d} i_{q 0} \\ -1 & 0 & 0 & 0 & 0 & 0 \\ 0 & 0 & 0 & 0 & 0 & -i_{q 0} \\ -K_{p s} & K_{i s} & 0 & 0 & 0 & i_{d 0} \\ 0 & 0 & K_{i d} & 0 & 0 & -K_{p d} i_{q 0} \\ 1 & 0 & 0 & 0 & 0 & 0\end{array}\right]$

$\boldsymbol{A}_{\boldsymbol{x}}=\left[\begin{array}{ccc}\omega_{c} K_{p e} K_{p d} & 0 & 0 \\ 0 & 0 & 0 \\ -1 & 0 & 0 \\ 0 & -1 & 0 \\ -K_{p d} & 0 & 0 \\ 0 & 0 & -1\end{array}\right], \quad \boldsymbol{B}_{\boldsymbol{r}}=\left[\begin{array}{cc}-\omega_{c} K_{p e} K_{p d} & 0 \\ 0 & 1 \\ 1 & 0 \\ 0 & K_{p s} \\ K_{p d} & 0 \\ 0 & 0\end{array}\right]$

$\boldsymbol{F}_{\boldsymbol{w}}=\left[\begin{array}{ccccc}-L_{q}^{*} i_{\delta 0} & 0 & K_{i d} & 0 & 0 \\ -K_{p q} K_{p s} & K_{p q} K_{i s} & 0 & K_{i q} & 0\end{array}\right.$ *

$$
\left.* \begin{array}{c}
-i_{q 0} K_{p d}+L_{q}^{*} \hat{\omega}_{r 0} i_{d 0}-v_{\delta 0}^{*} \\
K_{p q} i_{d 0}+v_{\gamma 0}^{*}
\end{array}\right]
$$

$\boldsymbol{F}_{\boldsymbol{x}}=\left[\begin{array}{ccc}-K_{p d} & -L_{q}^{*} \hat{\omega}_{r 0} & 0 \\ 0 & -K_{p q} & 0\end{array}\right], \quad \boldsymbol{F}_{\boldsymbol{r}}=\left[\begin{array}{cc}K_{p d}+R_{s}^{*} & 0 \\ 0 & K_{p q} K_{p s}\end{array}\right]$

Mineo Tsuji (Senior Member) was born in December 1953. He re-

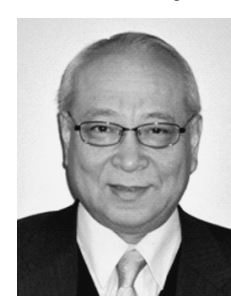
ceived the Ph.D. degree from Kyushu University in March 1981 in electrical engineering, became a lecturer at Nagasaki University in April 1981 and became a Professor in July 2001 in the Division of Electrical Engineering and Computer Science, Graduate School of Engineering, Nagasaki University. His current interest is control of $\mathrm{AC}$ machines and electrical power systems. Dr. Tsuji is a member of IEEE. He received a Prize Paper Award from the IEEJ in 1998.
Kazuya Kojima (Student Member) was born in April 1987. He re-

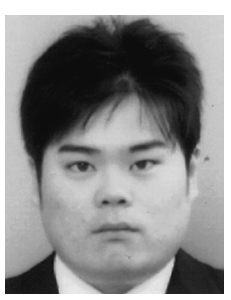
ceived the B.S. degree in electrical engineering from Nagasaki University in 2010. Since same year, he has entered a master's course of Faculty of Engineering in Nagasaki University Graduate School of Science and Technology. In 2012, he joined Mazda Motor Corporation. His research interest is about the sensorless vector control of IPMSM.

Glanny M. Ch. Mangindaan (Student Member) was born in March

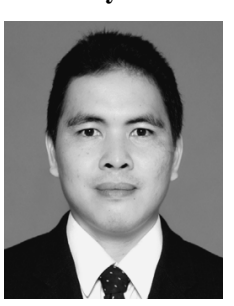
1974. He received the B.Eng. degree in electrical engineering from Sam Ratulangi University in 2000 and M.Eng. degree in electrical engineering from Sepuluh Nopember Institute of Technology in 2008. He became a lecturer in Division of Electrical Engineering at Sam Ratulangi University in December 2002. He has entered as Ph.D. course in Nagasaki University Graduate School of Engineering in 2012. His research interest is about the speed sensorless vector

control of AC motors.

Daisuke Akafuji (Student Member) was born in October 1988. He re-

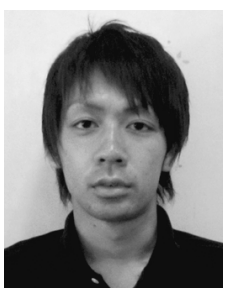
ceived the B.S. degree in electrical engineering from Nagasaki University in 2011. Since same year, he has entered a master's course of Faculty of Engineering in Nagasaki University Graduate School of Science and Technology. His research interest is about the sensorless vector control of IPMSM.

Shin-ichi Hamasaki (Member) was born in October, 1975. He re-

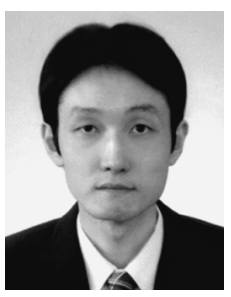
ceived the B.E. and M.E. and Ph.D. degrees in electrical engineering from Yokohama National University in 1998, 2000 and 2003 respectively. From 2003 to 2007, he was a Research Associate in the Department of Electrical and Electronic Engineering, Nagasaki University. Since April 2007, he has been an Assistant Professor in the Division of Electrical Engineering and Computer Science, Graduate School of Engineering, Nagasaki University. His current research interests are power conditioning systems and motor drives. 